\title{
Should children with inherited metabolic disorders receive varicella vaccination?
}

\author{
M Varghese, ${ }^{1}$ M Cafferkey, ${ }^{2}$ M O'Regan, ${ }^{1}$ A A Monavari, ${ }^{1}$ E P Treacy ${ }^{1}$
}

${ }^{1}$ National Centre for Inherited Metabolic Disorders, and

${ }^{2}$ Department of Clinical Microbiology, Children's University Hospital, Temple Street, Dublin, Ireland

\section{Correspondence to}

Professor E P Treacy, National Centre for Inherited Metabolic Disorders, Children's University Hospital, Temple Street, Dublin 1, Ireland; eileen.treacy@cuh.ie

Accepted 18 September 2009 Published Online First 21 October 2009

\section{ABSTRACT}

The aim was to determine the rate of varicella infection and complications in children with disorders of intermediary metabolism (IEM) between the ages of 1 and 16 years attending our national metabolic referral centre. Of 126 children identified, a response was received from 122. A history of previous varicella infection was identified in 64 cases (53\%) and of varicella vaccination in 5 (4\%). Fifty-three (43\%) patients apparently did not have a history of clinical varicella infection. Of the 64 children with a history of varicella infection, five required hospitalisation for complications, including life-threatening lactic acidosis in one patient with mitochondrial disease and metabolic decompensation in four patients. In conclusion, varicella infection may cause an increased risk of metabolic decompensation in patients with IEMs. We propose that a trial of varicella vaccination be considered for this cohort of patients with monitoring of its safety and efficacy.

Communicable infectious diseases of childhood place children with inborn errors of metabolism (IEMs) at high risk of metabolic decompensation with potentially catastrophic consequences. A number of IEMs also have an associated immunodeficiency. ${ }^{1}$ Varicella is an acute, highly communicable viral disease with worldwide distribution for which a preventive vaccination is available. In the USA, more than $90 \%$ of infections and two thirds of varicella-related hospitalisations and almost half of varicella-related deaths occur in children. ${ }^{2}$ Infection may also be fatal in immunocompromised individuals. Complications include secondary bacterial infections commonly by Streptococcus pyogenes and/or Staphylococcus aureus. ${ }^{2}$ Other complications include postinfectious cerebellar ataxia, encephalitis and respiratory complications, including pneumonitis.

Varicella vaccine is a live attenuated OKA strain and has been marketed since 1974, with two products now available, the univalent Varicella Virus Vaccine Live (VARIVAX) and multivalent ProQuad (MMR + varicella). ${ }^{3}$ Varicella vaccine is considered to be $85-90 \%$ effective in preventing varicella infection and $100 \%$ effective in preventing moderate or severe disease. ${ }^{2}$ Seroconversion after a single dose can reach up to $95 \%$ in healthy children. The US Advisory Committee on Immunization Practices (2006) now recommends two doses, the first at $12-15$ months and the second at 4-6 years. ${ }^{3}$

We sought to determine the rate of varicella infection and complications in the cohort of patients with IEMs actively followed at our centre, the National Centre for Inherited Metabolic Diseases.

\section{METHODS}

Patients with inborn errors of intermediary metabolism aged between 1 and 16 years on 1 July 2007 were identified from the National Centre for Inherited Metabolic Disorders database. Patients with Phenylketonuria, Galactosaemia, Homocystinuria and other conditions not associated with major metabolic decompensation during an acute illness were excluded from this study. A questionnaire was mailed to parents to ascertain whether the child had a prior history of varicella infection, any complications following varicella and/or history of varicella vaccination prior to the study.

\section{RESULTS}

We identified 126 patients at risk for metabolic decompensation, to whom the questionnaire was mailed. Non-responders were contacted by telephone. The total response rate was 97\% (52\% by post and $45 \%$ by phone). There was a history of varicella in $64(53 \%)$ patients. Five patients had previously been vaccinated. Fifty-three $(43 \%)$ patients did not have a history of clinical varicella infection. Of a subset of patients ${ }^{4}$ who had varicella serology recently performed and who were deemed from the history not to have been infected, three were found to be positive for varicella antibody. The characteristics of the study cohort and complications documented are described in table 1. Five children were hospitalised with varicella; all had metabolic decompensation on admission and needed intensive treatment. One child who had life-threatening lactic acidosis required intensive care. In addition, four patients with mitochondrial disease developed secondary bacterial skin infections. All skin infections were treated by family doctors, and hospitalisation was not required.

\section{DISCUSSION}

Our results indicate that the hospitalisation rate observed following varicella in patients with IEMs was at least $5 \%$ in the total group potentially exposed to varicella $(7.8 \%$ in the respondent group), which is much higher than the complication rate of $0.01 \%$ reported in apparently healthy children. ${ }^{5}$

Even allowing for the fact that some children whose families reported no history of varicella may have had subclinical or mild varicella, this hospitalisation rate is still significantly higher 
Table 1 Complications of varicella in children with inborn errors of metabolism

\begin{tabular}{lllll}
\hline Inborn errors of metabolism & Number & $\begin{array}{l}\text { History of clinical } \\
\text { varicella }\end{array}$ & $\begin{array}{l}\text { Metabolic decompensation } \\
\text { needing hospital admission }\end{array}$ & $\begin{array}{l}\text { Secondary } \\
\text { bacterial infection }\end{array}$ \\
\hline $\begin{array}{l}\text { Mitochondrial respiratory chain } \\
\text { defect }\end{array}$ & 53 & 29 & $2^{*}$ & 5 \\
Fatty acid oxidation disorder & 13 & 10 & 1 \\
Glycogen storage disorder & 9 & 2 & & \\
Urea cycle disorder & 9 & 6 & 1 \\
Glutaric aciduria & 8 & 5 & 1 \\
Mucopolysaccharidosis & 7 & 3 & \\
Maple syrup urine disease & 6 & 0 & \\
Methylmalonic aciduria & 5 & 3 & 6 & \\
Others & 12 & 6 &
\end{tabular}

* One child admitted to intensive care with lactic acidosis.

than for normal healthy children. The rate of bacterial skin infections observed in the cohort was similar to that for normal healthy children. ${ }^{4}$ There is little data on the complications of varicella in children with IEM. Hospitalisation from varicella in our patients with IEMs was all due to metabolic decompensation, not secondary bacterial infection (in contrast to other children). 56

Management of our patients typically included administration of "unwell dietary regimens".

We propose that varicella vaccination in children with IEMs shown by serology to be varicella negative may be beneficial. Follow-up with monitoring of patient seroconversion rates and documentation of any adverse events following vaccination will be required. Potential drawbacks from giving varicella vaccine to children with IEMs are metabolic decompensation and contraindication due to immunodeficiency. Fifteen per cent of children develop a fever following varicella vaccination, ${ }^{23}$ so they might be at risk of metabolic decompensation. However, our practice is to give an "unwell regime" with extra calories (or decreased protein intake in the case of organic acidurias) prophylactically at the time of vaccination and as required if there is a fever following vaccination.

Vaccination with varicella vaccine, a live vaccine, may be contraindicated in metabolic diseases associated with a significant immunodeficiency such as Glycogen Storage Disorder $1 \mathrm{~b}$ and poorly controlled propionic and methylmalonic aciduria; each case should be evaluated individually. ${ }^{1}$

In conclusion, varicella infection may cause an increased risk of metabolic decompensation in patients with IEMs. We propose that a trial of varicella vaccination is considered for this cohort of patients with monitoring of its safety and efficacy.

Acknowledgements Angela Hayes, RN, is thanked for her assistance with this study.

Competing interests None.

Provenance and peer reviewed Not commissioned; externally peer reviewed.

Patient consent Obtained.

\section{REFERENCES}

1. Kingsley JD, Varman M, Chatterjee A, et al. Immunizations for patients with metabolic disorders. Pediatrics 2006;118:e460-70.

2. American Academy of Pediatrics, Committee on Infectious Diseases. Varicella vaccine update. Pediatrics 2000;105;136-41.

3. Marin M, Guris D, Cahves S, et al. Prevention of varicella. Recommendations of the Advisory Committee on Immunization Practices (ACIP). MMWR 2007;56:1-42.

4. Somekh E, Dalal I, Shohat T, et al. The burden of uncomplicated cases of chickenpox in Israel. J Infect 2002;45:233-6.

5. Ziebold C, von Kries R, Lang R, et al. Severe complications of varicella in previously healthy children in Germany: a 1-year survey. Pediatrics 2001;108:e79.

6. Cameron JC, Allan G, Johnston F, et al. Severe complications of chickenpox in hospitalised children in the UK and Ireland. Arch Dis Child 2007;92:1062-6. 